Universidade Tecnológica Federal do Paraná - UTFPR

Campus Ponta Grossa - Paraná - Brasil

ISSN: $1981-3686$ / v. 02, n. 2: p. 25-31, 2008
Revista Brasileira de Tecnologia
Agroindustrial

\title{
AVALIAÇÃO DA QUALIDADE MICROBIOLÓGICA DE QUEIJO TIPO MUSSARELA COMERCIALIZADO NA CIDADE DE PONTA GROSSA, PARANÁ
}

\section{EVALUATION OF QUALITY OF MICROBIOLOGICAL MOZZARELLA CHEESE KIND OF TOWN MARKETED IN PONTA GROSSA, PARANÁ}

\author{
Giovana de Arruda Moura Pietrowski ${ }^{1}$, Marly Ranthum ${ }^{2}$; Talita Crozeta ${ }^{3}$; Vannessa de Jonge ${ }^{4}$ \\ 1,2,3,4 Universidade Tecnológica Federal do Paraná - UTFPR - Ponta Grossa - Brasil \\ giovana@utfpr.edu.br
}

\section{Resumo}

Queijo mussarela é um produto de fácil aceitação e grande rendimento na fabricação, porém muito suscetível á contaminação microbiológica. Este trabalho teve como objetivo analisar a qualidade microbiológica do queijo mussarela vendido em supermercados da cidade de Ponta Grossa, PR. Foram realizadas as análises microbiológicas recomendadas pela $R D C n^{o}$. 12, de 2 de janeiro de 2001: contagem de Coliformes fecais $\left(45^{\circ} \mathrm{C}\right)$, Salmonella sp. e Estafilococos coagulase positiva. Os resultados mostraram que todas as amostras apresentaram-se dentro dos padrões para Coliformes Fecais, uma amostra (6,25\%) apresentou presença de Salmonella sp., e três amostras (18,75\%) apresentaram-se fora dos padrões para Estafilococos coagulase positiva. Com estes resultados pode-se afirmar que algumas amostras estão impróprias para o consumo humano, e que a fiscalização nos mercados de Ponta Grossa, e nos fabricantes que fornecem o produto para a região deve ser mais eficiente.

Palavras-chave: qualidade microbiológica, queijo; mussarela.

\section{Introdução}

Segundo o Ministério da Agricultura e do Abastecimento, entende-se por queijo o produto fresco ou maturado obtido por separação parcial do soro do leite ou leite reconstituído (integral, parcial, ou totalmente desnatado), ou de soros lácteos coagulados pela ação física do coalho, de enzimas específicas, de bactérias específicas, de ácidos orgânicos, isolados ou combinados, todos de 
qualidade aceitável para o uso alimentar, com ou sem agregação de substâncias alimentícias e/ou condimentos, aditivos especificamente indicados, substâncias aromatizantes e matérias corantes (BRASIL, 2008).

A fabricação de queijos consiste uma série de operações desde a produção de leite até o último dia de maturação e expedição para o mercado. A qualidade do queijo depende diretamente da qualidade do leite, sendo necessário um rígido controle de qualidade durante todas as fases de processamento, contudo, o queijo é um alimento de grande comercialização, apresentando vantagens do ponto de vista tecnológico: é um produto de fácil aceitação, apresenta elevado rendimento na fabricação, o que implementa o seu escoamento e distribuição no mercado (FURTADO, 1991).

O queijo Mussarela é um queijo suave, de massa macia, com fabricação concentrada no sul da Itália, principalmente. Sua fabricação iniciou-se por volta do século XVI, era produzido exclusivamente a partir do leite de búfala, mas devido a grande escassez ocorreu a mistura com o leite de vaca ou ainda era produzido exclusivamente a partir do leite de vaca. Para ALBUQUERQUE (2003), uma boa Mussarela deve possuir uma crosta fina, consistência semi-sura, textura compacta, fechada, coloração esbranquiçada, odor suave e sabor ligeiramente ácido. Deve apresentar também, um rendimento entre 9,5 a 10,5 litros de leite por quilo de queijo, dependendo da composição do leite, enquanto que para consumo direto este rendimento pode ser melhor.

O queijo é composto por 46 a 49\%, de umidade, segundo ALBUQUERQUE (2003), proveniente do leite, sendo alvo dos microrganismos, que utilizam esta água para aumentar a atividade água do meio, favorecendo a sua multiplicação. Apresenta 22 a $24 \%$ de gordura, pH entre 5,1 e 5, 3, um pouco ácido que favorece o crescimento de alguns microrganismos, quantidade de sal de1,6 a 1,8\%, proteínas e lactose.

Todos estes nutrientes favorecem a proliferação de microrganismos potógenos que são de grande risco à saúde humana. Estes nutrientes são fontes para que eles se desenvolvam e a comercialização do produto em desacordo com os padrões de qualidade microbiológica vigentes pode refletir na ocorrência de casos e surtos de doenças transmitidas por alimentos, o que aumenta a preocupação com as características microbiológicas do produto. Os coliformes fecais também chamados de microrganismos indicadores, são grupos ou espécies de microrganismos que quando estão presentes no alimento e fornecem informações sobre a ocorrência de contaminação de origem fecal e a provável presença de microrganismos patogênicos. Vivem no intestino de animais de sangue quente e têm como principal representante a Escherichia coli, que segundo FORSYTHE (2002) se desenvolvem em atividade água de no mínimo 0,935, pH em torno de 4,0 - 9,0 e faixa de temperatura $7^{\circ}$ a $49,4^{\circ} \mathrm{C}$. 
As bactérias do gênero Salmonella sp. são patogênicas e são responsáveis pela maior causa de surtos de toxinfecções alimentares. A contaminação ocorre devido ao controle inadequado de temperatura, de práticas de manipulação incorretas ou por contaminação cruzada de alimentos crus com alimentos processados. Sua simples presença no alimento implica na rejeição de todo lote, por isso a análise de Salmonella sp. é dita qualitativa (FRANCO e LANDGRAF , 2002).

Para FRANCO E LANDGRAF, a presença de números elevados de Staphylococcus aureus é uma indicação de perigo potencial à saúde pública devido à enterotoxina estafilocócica presente no alimento, bem como à sanificação questionável, principalmente quando o processo envolve manipulação do alimento, por ser um microrganismo que vive nas mucosas e na pele. Apresentam atividade de água mínima de $0,83, \mathrm{pH}$ entre 4 e 10 e a faixa de temperatura de $7^{\circ}$ a $50^{\circ} \mathrm{C}$ (FORSYTHE, 2002).

Diante do exposto, o presente trabalho teve como objetivo avaliar a qualidade microbiológica do queijo tipo mussarela fatiado, comercializado na região de Ponta Grossa/PR, por meio da detecção de Salmonella sp; contagem de Coliformes a $45^{\circ} \mathrm{C}$ e Contagem de Estafilococos coagulase positiva, confrontando os resultados obtidos com os padrões legais vigentes.

\section{Material e métodos}

Como quesito parcial de avaliação da disciplina de Microbiologia Aplicada, do Curso Superior de Tecnologia de Alimentos, foram coletadas e analisadas 16 amostras de queijo tipo mussarela fatiado, de diferentes marcas, em supermercados da cidade de Ponta Grossa, Paraná. Estas amostras foram transportadas sob refrigeração em caixas isotérmicas contendo gelo, até o Laboratório de Microbiologia da Universidade Tecnológica Federal do Paraná - Campus Ponta Grossa.

Foram realizadas as análises microbiológicas recomendadas pela $\mathrm{RDC} \mathrm{n}^{\mathrm{o}}$. 12, de 2 de janeiro de 2001: contagem de Coliformes fecais $\left(45^{\circ} \mathrm{C}\right)$, Salmonella sp. e Estafilococos coagulase positiva, todas realizadas de acordo com o Manual de Métodos de Análise Microbiológica de Alimentos SILVA et al (1997).

Cada amostra foi pesada assepticamente (25g da amostra) e feita a diluição $10^{-1}$ em água salina peptonada a $0,1 \%$, e a partir desta primeira diluição preparou-se as outras diluições, $10^{-2}, 10^{-3}$ e $10^{-4}$.

Para a análise de Coliformes fecais, as diluições foram semeadas em quatro placas de Petri 
contendo Ágar Vermelho Violeta Bile (VRBA), por plaqueamento direto Pour Plate e incubou-se as placas em estufa com temperatura de $45^{\circ} \mathrm{C}$ de 24 horas a 48 horas, invertendo as placas. Após as 48 horas, retirou-se as placas da estufa e realizou-se a contagem de colônias típicas.

Para a análise de Salmonella sp. utilizou-se como caldo de enriquecimento não seletivo a água destilada peptonada tamponada a $1 \%$ (ADPT), onde pesou-se $25 \mathrm{~g}$ da amostra e incubou-se a $35^{\circ} \mathrm{C}$ por 24 horas. Após a incubação, transferiu-se $1 \mathrm{~mL}$ da cultura obtida para um tubo de Caldo Rappaport e 0,2 mL em outro tubo com o mesmo caldo, incubando-se à temperatura de $41^{\circ} \mathrm{C}$ por 24 horas. A seguir, realizou-se plaqueamento seletivo-diferencial em Ágar Salmonella-Shigella (SS) e Ágar Verde Brilhante (BGA), por estriamento descontínuo. Após incubação a $35^{\circ} \mathrm{C}$ por 24 horas, verificou-se o aparecimento de colônias típicas e procedeu-se os testes bioquímicos. Utilizou-se Ágar Tríplice Açúcar Ferro (TSI) e Ágar Lisina Ferro (LIA), os quais foram semeados com fio de platina, inoculados com uma colônia típica e bem isolada. Incubou-se a $35^{\circ} \mathrm{C}$ por 24 horas e anotou-se os resultados obtidos.

Para a análise Estafilococos Coagulase Positiva, as diluições foram semeadas em superfície com alça de Drigalski em Ágar Baird-Parker, suplementado com emulsão de gema de ovo e solução de Telurito de Potássio. Incubou-se as placas a $35^{\circ} \mathrm{C}$ por $48 \mathrm{~h}$, invertendo-se as placas. Após as $48 \mathrm{~h}$, retirou-se as placas da estufa contou-se as colônias típicas de Estafilococos. Isolou-se 1 colônia de cada placa para realizar a prova da coagulase.

\section{Resultados e discussões}

Os resultados das análises microbiológicas realizadas estão registrados na Tabela 1.

Tabela 1 - Resultados das Análises Microbiológicas

\begin{tabular}{|c|c|c|c|}
\hline & Coliformes $^{4} 5^{\circ} \mathbf{C}$ & Salmonella sp. & $\begin{array}{c}\text { Estafilococos } \\
\text { Coagulase Positiva }\end{array}$ \\
\hline Amostra 1 & $<10 \mathrm{UFC} / \mathrm{g}$ & Ausência em $25 \mathrm{~g}$ & $<10 \mathrm{UFC} / \mathrm{g}$ \\
\hline
\end{tabular}




\begin{tabular}{|c|c|c|c|}
\hline Amostra 2 & $<10 \mathrm{UFC} / \mathrm{g}$ & Presença em $25 \mathrm{~g}$ & $7 \times 10 \mathrm{UFC} / \mathrm{g}$ \\
\hline Amostra3 & $1 \times 10^{3} \mathrm{UFC} / \mathrm{g}$ & Ausência em $25 \mathrm{~g}$ & $10^{3} \mathrm{UFC} / \mathrm{g}$ \\
\hline Amostra 4 & $<10 \mathrm{UFC} / \mathrm{g}$ & Ausência em $25 \mathrm{~g}$ & $<10 \mathrm{UFC} / \mathrm{g}$ \\
\hline Amostra 5 & $<10 \mathrm{UFC} / \mathrm{g}$ & Ausência em $25 \mathrm{~g}$ & $<10 \mathrm{UFC} / \mathrm{g}$ \\
\hline Amostra 6 & $<10 \mathrm{UFC} / \mathrm{g}$ & Ausência em $25 \mathrm{~g}$ & $<10 \mathrm{UFC} / \mathrm{g}$ \\
\hline Amostra 7 & $<10 \mathrm{UFC} / \mathrm{g}$ & Ausência em $25 \mathrm{~g}$ & $2 \times 10^{4} \mathrm{UFC} / \mathrm{g}$ \\
\hline Amostra 8 & $<10 \mathrm{UFC} / \mathrm{g}$ & Ausência em $25 \mathrm{~g}$ & $<10 \mathrm{UFC} / \mathrm{g}$ \\
\hline Amostra 9 & $<10 \mathrm{UFC} / \mathrm{g}$ & Ausência em $25 \mathrm{~g}$ & $3 \times 10^{4} \mathrm{UFC} / \mathrm{g}$ \\
\hline Amostra 10 & $<10 \mathrm{UFC} / \mathrm{g}$ & Ausência em $25 \mathrm{~g}$ & $<10 \mathrm{UFC} / \mathrm{g}$ \\
\hline Amostra 11 & $<10 \mathrm{UFC} / \mathrm{g}$ & Ausência em $25 \mathrm{~g}$ & $8,4 \times 10^{4} \mathrm{UFC} / \mathrm{g}$ \\
\hline Amostra 12 & $>10 \mathrm{UFC} / \mathrm{g}$ & Ausência em $25 \mathrm{~g}$ & $10^{2} \mathrm{UFC} / \mathrm{g}$ \\
\hline Amostra 13 & $<10 \mathrm{UFC} / \mathrm{g}$ & Ausência em $25 \mathrm{~g}$ & $<10 \mathrm{UFC} / \mathrm{g}$ \\
\hline Amostra 14 & $<10 \mathrm{UFC} / \mathrm{g}$ & Ausência em $25 \mathrm{~g}$ & $<10 \mathrm{UFC} / \mathrm{g}$ \\
\hline Amostra 15 & $<10 \mathrm{UFC} / \mathrm{g}$ & Ausência em $25 \mathrm{~g}$ & $8,0 \times 10^{2} \mathrm{UFC} / \mathrm{g}$ \\
\hline Amostra 16 & $<10 \mathrm{UFC} / \mathrm{g}$ & Ausência em $25 \mathrm{~g}$ & $9,0 \times 10^{2} \mathrm{UFC} / \mathrm{g}$ \\
\hline $\begin{array}{l}\text { PADRÃO DA } \\
\text { RDC 12/2001 }\end{array}$ & $5 \times 10^{3} \mathrm{UFC} / \mathrm{g}$ & Ausência em 25g & $10^{3} \mathrm{UFC} / \mathrm{g}$ \\
\hline
\end{tabular}

De acordo com normas vigentes da Agência Nacional de Vigilância Sanitária (ANVISA), a Resolução RDC n 12 de 02 de janeiro de 2001 estabelece um valor limite para cada microrganismo analisado. Neste sentido, os resultados das análises para Coliformes $45^{\circ} \mathrm{C}$ revelaram que $100 \%$ das amostras encontra-se dentro do padrão, que para este microrganismo é de $5 \times 10^{3} \mathrm{UFC} / \mathrm{g}$.

No entanto, RIBEIRO (2003) não confirma esses resultados, já que uma amostra das 21 amostras analisadas encontrava-se fora do referido padrão, isso pode significar manipulação sem higiene, armazenamento inadequado, recontaminação pós-processamento ou ainda contaminantes provenientes da matéria-prima (FRANCO e LANDGRAF , 2002).

MONTEIRO e BADARÓ (2006) tendo analisado 15 amostras de queijo tipo minas frescal na cidade de Ipatinga (MG), verificaram a presença de Coliformes a $45^{\circ} \mathrm{C}$ em todas as amostras analisadas, sendo que $60 \%$ estavam fora dos padrões legais vigentes.

Nas análises realizadas para Salmonella sp., umas das amostras $(6,25 \%)$ estava fora do padrão. Este resultado não é confirmado por RIBEIRO (2003) que obteve 100\% de suas amostras dentro do padrão vigente e também não é confirmado por OLIVIERI (2004), que não detectou a presença do mesmo microrganismo em nenhuma das amostras de mussarela fabricada a partir de leite de búfala que analisou.

$\mathrm{Na}$ análise de Estafilococos Caogulase Positiva, três amostras (18,75\%) encontraram-se acima do padrão estabelecido pela RDC 12/2001, que para este microrganismo é de $10^{3} \mathrm{UFC} / \mathrm{g}$. Este resultado é similar ao encontrado por SILVA et al (apud RIBEIRO, 2003) que verificaram presença de S. aureus em $27 \%$ das 30 amostras analisadas, e também para MONTEIRO e 
BADARÓ (2006) que nas análises de Staphylococcus, encontraram 92,8\% dos resultados positivos para o teste de coagulase.

No entanto, não é confirmado por RIBEIRO (2003) que verificou $100 \%$ de suas amostras dentro do padrão e também por QUINTANA e CARNEIRO (2007) que tendo analisado queijo tipo Mussarela e Minas Frescal, da cidade de Morrinhos em Goiás, nos meses de abril, junho, julho e agosto, na contagem de Staphylococcus coagulase positiva, evidenciou que apenas o resultado obtido no mês de maio, e para o queijo tipo Minas Frescal, apresentou valores acima do limite estabelecido pela legislação.

\section{Conclusão}

Os resultados encontrados nas análises revelaram que algumas amostras demonstraram características impróprias para o consumo humano, para Salmonella sp e Estafilococos coagulase positiva, por apresentarem uma contagem significativa destes microrganismos e acima do padrão vigente na legislação.

Entretanto, como as amostras coletadas foram do queijo mussarela fatiado, não se pode afirmar que a contaminação é do próprio produto ou se ocorreu no processo de fatiamento do mesmo. Assim, percebe-se a necessidade de realizar uma pesquisa do queijo mussarela, com amostras não fatiadas e fatiadas de um mesmo lote do produto, para que o problema de contaminação possa ser rastreado e resolvido.

De qualquer forma fica um alerta para uma maior fiscalização nos mercados da cidade e também para os fabricantes da mussarela comercializada na região de Ponta Grossa, para que o consumidor possa utilizar produtos com qualidade microbiológica adequada, preservando sua saúde e bem estar.

\section{Abstract}

Mozzarella cheese is a product of easy acceptance and high yield in manufacturing; however very susceptible á microbiologycal contamination. This study aimed to examine the microbiological quality of mozzarella cheese sold in supermarkets in the city of Ponta Grossa, PR. Were conducted microbiological analyses recommended by DRC paragraph. 12, January 2, 2001: counting of fecal coliforms (45 C), Salmonella species. and Estafilococos coagulase positive. The results showed that all samples submitted within the standards for Fecais coliforms, a sample (6.25\%) showed presence of Salmonella sp., And three samples (18.75\%) showed up outside of the standards for Estafilococos coagulase positive. With these results can be said that some samples are unfit for human consumption, and that the surveillance in the markets of Ponta Grossa, and the 
manufacturers who supply the product to the region should be more efficient.

Key-words: microbiological quality; cheese; mozzarella

\section{Referências}

BRASIL. Ministério da Agricultura, Pecuária e Abastecimento. Disponível em <http:// www.agricultura.gov.br/das/req.queijomozzarella.htm .> Acesso em 5 abr, 2008.

Agencia Nacional de Vigilância Sanitária (ANVISA). Resolução RDC no 12 de 02 Janeiro de 2001. Disponível em: $<$ http://www.anvisa.gov.br> Acesso em 15 abr 2008.

FURTADO, M. M. A arte e a ciência do queijo. 2. ed. São Paulo: Globo. 1991.

ALBUQUERQUE, L. C. de. Os queijos no mundo. Juiz de Fora: ILCT, 2002-2003. 5. v.

FRANCO, B. D. G. de M.; LANDGRAF, M. Microbiologia dos Alimentos. São Paulo: Editora Atheneu, 2002.

FORSYTHE, S. J. Microbiologia da Segurança Alimentar. Porto Alegre: Artmed, 2002.

RIBEIRO, C. DE C. Análise da Qualidade Microbiológica do Queijo Mussarela Fatiado Disponível em Supermercados da Cidade de Ponta Grossa, no Estado do Paraná. Ponta Grossa: 2003.

MONTEIRO, M; V.; BADARÓ, A. C. L. Qualidade Microbiológica de Queijos tipo Minas Frescal comercializados na cidade de Ipatinga, Minas Gerais. Revista Higiene Alimentar. São Paulo, v. 21, n. 150, p. 230-231, 2006.

QUINTANA, R. C.; CARNEIRO, L. C. Avaliação das condições higiênico-sanitárias dos queijos minas frescal e mussarela produzidos na cidade de Morrinhos - GO. Revista Brasileira de Saúde e Produção Animal. v. 8, n.3, p. 205-211, 2007. Disponível em: <http://www.rbspa.ufba.br> Acesso em 15 abr 2008.

OLIVIERI, D. A. Avaliação da Qualidade Microbiológica de amostras de mercado de Queijo Mussarela, elaborado a partir de leite de búfala. São Paulo: 2004.

Nome completo: Giovana de Arruda Moura Pietrowski

Filiação institucional: Universidade Tecnológica Federal do Paraná

Departamento: Tecnologia de Alimentos

Função ou cargo ocupado: Professora Dedicação exclusiva

Telefones para contato: (42) 3220-4823

e-mail: giovana@utfpr.edu.br 